\title{
Water thermoporosimetry as a tool of characterization of the textural parameters of mesoporous materials
}

\author{
Refinement of the methodology
}

\author{
D. Majda ${ }^{1}$ M. Zimowska ${ }^{2}$ K. Tarach ${ }^{1}$ K. Góra-Marek ${ }^{1}$ B. D. Napruszewska ${ }^{2}$. \\ A. Michalik-Zym ${ }^{2}$
}

Received: 30 October 2015/Accepted: 11 March 2016/Published online: 28 March 2016

(c) The Author(s) 2016. This article is published with open access at Springerlink.com

\begin{abstract}
Water thermoporosimetry (TPM) is a powerful method for studying the properties of porous materials, devoted especially for investigation of the samples that can be destroyed in drying process. However, this method is not very popular and relatively rarely used because of problems with proper measurement procedure and choosing correct equation for the result interpretation. This report focused on refinement of general experimental protocols for water TPM. For the first time, the role of various parameters on the TPM outcomes was deeply investigated and simple and fast experimental mode was proposed. Additionally, based on the series of mesoporous silica SBA-15, the calibration procedure was employed and the reliable calibration equations were established.
\end{abstract}

Keywords Thermoporosimetry $\cdot$ Water $\cdot$ SBA-15 $\cdot$ Pore size distribution

\section{Introduction}

Due to increasing use of mesoporous materials, in various fields such as catalysis [1] adsorbents [2], electronics [3], drug delivery $[4,5]$ or medical diagnostics $[6]$, there is an

Electronic supplementary material The online version of this article (doi:10.1007/s10973-016-5400-3) contains supplementary material, which is available to authorized users.

D. Majda

majda@chemia.uj.edu.pl

1 Faculty of Chemistry, Jagiellonian University, Ingardena 3, 30-060 Kraków, Poland

2 Jerzy Haber Institute of Catalysis and Surface Chemistry, PAS, Niezapominajek 8, 30-239 Kraków, Poland increasing need for characterization of their porosity. The methods which are being considered as standard ones are mercury porosimetry and adsorption isotherms of nitrogen or argon $[2,7]$. Usually, their limitations are not taken into account. In mercury, porosimetry care must be taken that the sample is dry, because mercury will not displace liquid which is already present in the pores. This could be a disadvantage when characterizing hydrophilic samples. Moreover, high mercury pressures may crush or deform a pore. This concern is especially important when trying to accurately measure the pore volume of soft material, such as paper or membrane media [8, 9]. Gas adsorption porosimetry, operating on the principle of the micropore filling and capillary condensation in the mesopores, necessitates an understanding of the properties of the liquid phase confined inside the pores, which may be different from those of liquid adsorbate under standard conditions. However, in these methods, no influence of the pore curvature on the properties of the adsorbed phase is taken into consideration. The desorption isotherm used in the determination of the pore size distribution is affected by the pore network: When pressure is reduced, liquid will evaporate from large open pores, but pores of the same size that are connected to the surface with narrower channels remain filled [10]. This changes the shape of the pore size distribution. Despite the fact that this method has been used for decades, there are still some other effects that are not properly understood, e.g. hysteresis phenomena [11-13]. Disadvantages listed above as well as long-time sample preparation and measurements, expensive equipment, large amounts of samples needed and limited choice of the adsorptives are the main drawbacks of the standards method. Thermoporosimetry (TPM) seems to be a promising alternative, but it still needs further development. It is based on the fact that a fluid confined in the 
pores of a solid material experiences an important shift of its liquid-to-solid transition temperature $(\Delta \mathrm{T})$ and this shift is related to the size of the pores, in which the liquid is trapped [14], according to the Gibbs-Thomson Eq. (1):

$\Delta T=T-T_{0}=-\frac{\gamma_{l s} T_{0}}{\rho \Delta H} \frac{\mathrm{d} A}{\mathrm{~d} V}=-K \frac{\mathrm{d} A}{\mathrm{~d} V}$

where $\Delta T$ is the melting point depression, $T_{O}$ is the bulk melting temperature, $\gamma_{l s}$ is the surface tension of liquidsolid interface, $\rho$ is the density, $\Delta H$ is the specific enthalpy of melting, and $\mathrm{d} A / \mathrm{d} V$ is the curvature of the solid-liquid interface which is $1 / r$ for cylinders and $2 / r$ for spheres, where $r$ is the radius of the curvature [15]. Differential scanning calorimetry (DSC) is a technique proposed in 1955 by Kunh et al. $[16,17]$ to measure $\Delta T$. He discovered that soaking the porous material in the liquid and measuring the melting or crystallization profiles by DSC is enough to perform the porosity characterization. Moreover, it turned out that application of this technique is very simple, fast, inexpensive and non-destructive.

Several liquid compounds have been used to probe porous properties, for example benzene [15], acetonitrile [18], carbon tetrachloride [19], acetone [20] or n-alkanes $[21,22]$; however, water was historically the first and still remains the most common probe liquid. It is relevant especially for examining materials and coatings designed specifically to absorb aqueous solutions. It allows the measurement of moist samples, which is very advantageous for the investigation of pore of the materials that exist only in the swollen state [23]. On the other hand, water TPM seems to be a unique method for studying the porosity of the hydrated materials which can collapse during drying. Another advantage of using water is that its heat of fusion, $\Delta H_{\mathrm{m}}=334 \mathrm{Jg}^{-1}$, is up to an order of magnitude larger than the heat of fusion of most organic liquids. The large $\Delta H_{m}$ enhances the sensitivity of the DSC technique and allows decreasing size of the studied samples [24].

Despite simplicity and versatility of the DSC porosimetry, so far this method has been rarely used for routine characterization of the porous materials. A serious drawback in the TPM technique lies in the fact that the physical parameters of Gibbs-Thomson equation, such as surface tension, heat of fusion and density, must be known a priori. Also the temperature dependencies on these parameters should be known in the temperature range of the experiment. This is challenging if not impossible to determine them independently. Other complications arise from the persistence of an unfrozen adsorbed layer on the pore walls. It is well known that not all the solvent takes part in the phase transition and that a significant part of it is adsorbed on the surface of the pore and remains liquid. Consequently, the radius determined using the Gibbs-
Thomson equation does not reflect the real pore size. In order to avoid those problems, the experimental work often resorts to the use of reference materials with known pore size for calibration procedure. Since the TPM attracts more and more attention, correlation between pore radius and melting depression temperature, obtained from fitting a polynomial to the calibration data, for various liquids can be found in the references [15, 18, 22, 24]. In the case of water TPM, numerous studies have been performed, but choosing the right formula for the determination of the pore size is quite complicated. It is still not entirely clear why the equations for the same liquid given by various groups differ from each other. Probably, the reason is that they were not obtained by the same manner and the materials taken for calibration were also different, especially in the range of pore radii. Another reason can come from the fact that not all authors take into account the experimental parameters that may impact the TPM results. Quite common is also following the trend of linear dependence between $\Delta T$ on $1 / R$, although nonlinearity was empirically observed [25]. For better illustration of the problem of choosing the proper equations for TPM, the comparison of pore size distribution (PSD) profiles derived from water TPM experiments and calculated based on the different equations is shown in the Fig. 1.

In our previous papers, we focused on n-alkane TPM and we found out that experimental protocol is crucial in order to obtain reliable outcomes since various parameters can impact TPM experiments [21, 22]. The aim of this paper was to refine the water TPM method to make it more useful for wider spectrum of potential users by examining the influence of various parameters on the water TPM results, summarizing and simplifying the experimental protocol and establishing reliable calibration equations.

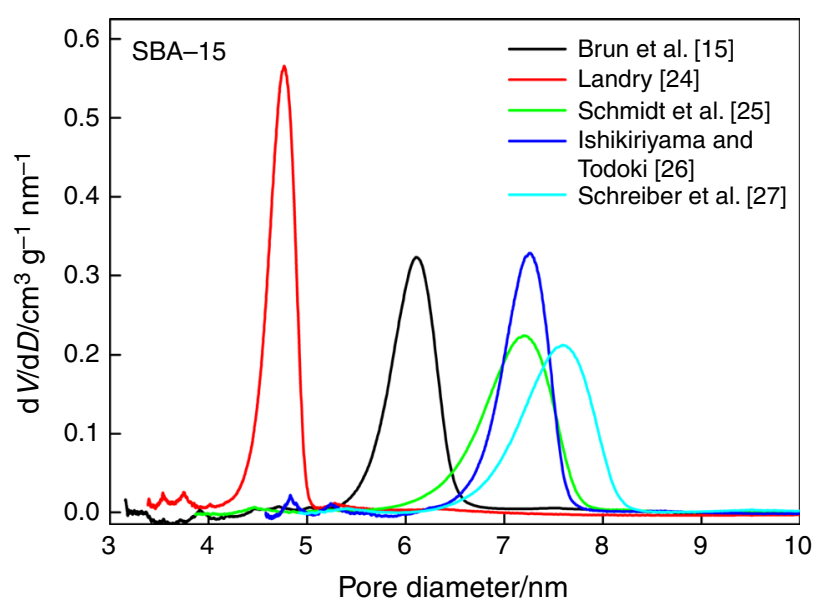

Fig. 1 Pore size distribution of the same SBA-15 sample derived from water TPM and calculated based on equation provided by various authors 
Series of ordered mesoporous silicas SBA-15, differing by the hexagonal unit cell parameter $a$, was chosen as model materials because of the ease in tailoring the uniform mesopores of various diameters and relatively thick silica walls which provide high stability and ensure long storage time [28]. Additionally, the method was checked by study porous properties of functionalized silica material obtained by anchoring of the N-[3-(trimethoksysilil)prophyl]etylenodiamine (AEAPTS) functional group on the internal and/ or external surface of SBA-15.

\section{Experimental}

Prior to the TPM measurements, based on DSC experiments, the samples were characterized by small angle $\mathrm{X}$-ray diffraction, electron microscopy (SEM/TEM) and low temperature $\mathrm{N}_{2}$ adsorption. Additionally, FT-IR technique was used to check the influence of the silanol groups presented in internal surface of SBA-15 on the TPM results.

\section{Materials}

The SBA-15 silicas were prepared according to the method described in our previous papers [22]. Briefly, the mixture of Pluronic P123 [(EO)20(PO)70(EO)20, Sigma-Aldrich], $\mathrm{H}_{2} \mathrm{O}, \mathrm{HCl}(37 \%)$ and TEOS [tetraethylorthosilicate Sigma-Aldrich], corresponding to a molar ratio of $1 \mathrm{SiO}_{2} /$ $0.017 \mathrm{P} 123 / 2 \mathrm{HCl} / 80 \mathrm{H}_{2} \mathrm{O}$, was added at $55^{\circ} \mathrm{C}$ for $24 \mathrm{~h}$ and then hydrothermally treated for the next $24 \mathrm{~h}$ either at $40,80,100$ or $150{ }^{\circ} \mathrm{C}$ and additionally for the next $72 \mathrm{~h}$ at $150{ }^{\circ} \mathrm{C}$. The products were dried at $80{ }^{\circ} \mathrm{C}$ and calcined at $550{ }^{\circ} \mathrm{C}$ for $5 \mathrm{~h}$ in air. The samples prepared at progressively increasing temperature of hydrothermal treatment are referred to as D1, D4 D5 and D6. D7 sample was obtained by prolonged hydrothermal treatment up to $72 \mathrm{~h}$ at $150{ }^{\circ} \mathrm{C}$. Silicas denoted D2 and D3 were prepared from the same initial mixture that was added at 30 or $35{ }^{\circ} \mathrm{C}$ for $24 \mathrm{~h}$ and subsequently treated hydrothermally for the next $24 \mathrm{~h}$ at $100{ }^{\circ} \mathrm{C}$.

To prepare functionalized SBA-15 silica, denoted as F1AEAPTS, first SBA-15 (F) was prepared from the same initial mixture that was added at $55^{\circ} \mathrm{C}$ for $24 \mathrm{~h}$ and subsequently treated hydrothermally for the next $24 \mathrm{~h}$ at $130{ }^{\circ} \mathrm{C}$. The above material was further calcined to remove the organic template. Before functionalization, the samples were exposed for $5 \mathrm{~h}$ to water vapour followed by drying for $2 \mathrm{~h}$ at $200{ }^{\circ} \mathrm{C}$. As conditioned, $1 \mathrm{~g}$ of SBA- 15 supports $(\mathrm{F})$ reacted with $20 \mathrm{~m} \mathrm{~mol} \mathrm{dm}^{-3}$ of $\mathrm{N}$-[3-(trimethoksysilil)prophyl]etylenodiamine (AEAPTS) diluted in toluene the sample was stirred under $40{ }^{\circ} \mathrm{C}$ for $20 \mathrm{~h}$. The resultant white solid (named F-AEAPTS) was filtered off and dried.

\section{XRD and SEM/TEM}

$\mathrm{X}$-ray diffraction patterns were recorded with a Philips 1710 powder diffractometer using Ni-filtered $\mathrm{Cu} \mathrm{K}_{\alpha}$ radiation. Morphology and structural ordering of the investigated materials were studied by means of JEOL JSM7500F Field Emission scanning electron microscope, using SEM and TEM modes.

\section{Nitrogen adsorption-desorption isotherms}

Specific surface area and pore size distribution were determined from the nitrogen adsorption-desorption isotherms obtained at $-196{ }^{\circ} \mathrm{C}$ with a Quantachrome Nova 2000 apparatus. Prior to the measurement, the samples were outgassed for $18 \mathrm{~h}$ at $200{ }^{\circ} \mathrm{C}$. Specific surface area determination was based on BET formalism. Pore size distribution analysis was carried out using the non-local density functional theory (DFT Kernel: $\mathrm{N}_{2}$ at $77 \mathrm{~K}$ on silica, cylindrical pore, NLDFT equilibrium model) which is recommended for the characterization of nanoporous materials [29]. The micropore volume was determined using the $t$-plot method.

\section{FT-IR}

The FT-IR spectra were recorded with a Bruker Tensor 27 spectrometer equipped with a MCT detector with the spectral resolution of $1 \mathrm{~cm}^{-1}$. Prior to FT-IR studies, all studied materials had been pressed into the form of selfsupporting discs (ca. 4-7 $\mathrm{mg} \mathrm{cm}^{-2}$ ), weighted and pretreated in situ in a home-made quartz IR cell at $350{ }^{\circ} \mathrm{C}$ under vacuum conditions for $30 \mathrm{~min}$. The portions of water (600-800 $\mu \mathrm{mol})$ have been introduced to IR quartz cell at room temperature up to the complete saturation of silanol groups. All the spectra presented in this work were normalized to $10 \mathrm{mg}$ of sample.

\section{TPM (DSC)}

The DSC measurements were performed using Mettler Toledo apparatus DSC $822^{\mathrm{e}}$ equipped with a liquid nitrogen cooling system allowing work between -150 and $400{ }^{\circ} \mathrm{C}$. Calibration for heat flux and temperature was done with an n-octane, indium and zinc standards. The melting point depression was determined relative to the excess phase, so that each experiment was internally calibrated for temperature [24]. Before the TPM experiment, a sample was placed in aluminium pan, the liquid was added, and the pan was sealed with lid. To avoid super-cooling effect, the samples were quenched far below the equilibrium freezing temperature. Pore size distribution was determined from the solid-to-liquid DSC profiles. Thermoporosimetry 
measurements were done with the temperature rate $\beta=2{ }^{\circ} \mathrm{C} \min ^{-1}$. After the TMP experiments, a small hole was made in the crucible's lid and the sample was heated up to $400{ }^{\circ} \mathrm{C}$ in order to evaporate the liquid component and determine its mass.

\section{Results and discussion}

XRD and SEM/TEM experiments confirmed presence of well-ordered hexagonal symmetry in all studied samples. Also, TEM images show parallel channels of dimensions increasing when passing from D1 to D7 samples. The pore size distributions as well as other porosity parameters obtained from $\mathrm{N}_{2}$ isotherms (Fig. 2; Table 1) are in agreement with typical characteristics expected for SBA15 silicas. The XRD patterns and SEM/TEM images were already published in our previous paper [22] and are additionally available as supplementary materials (S1 and S2, respectively).
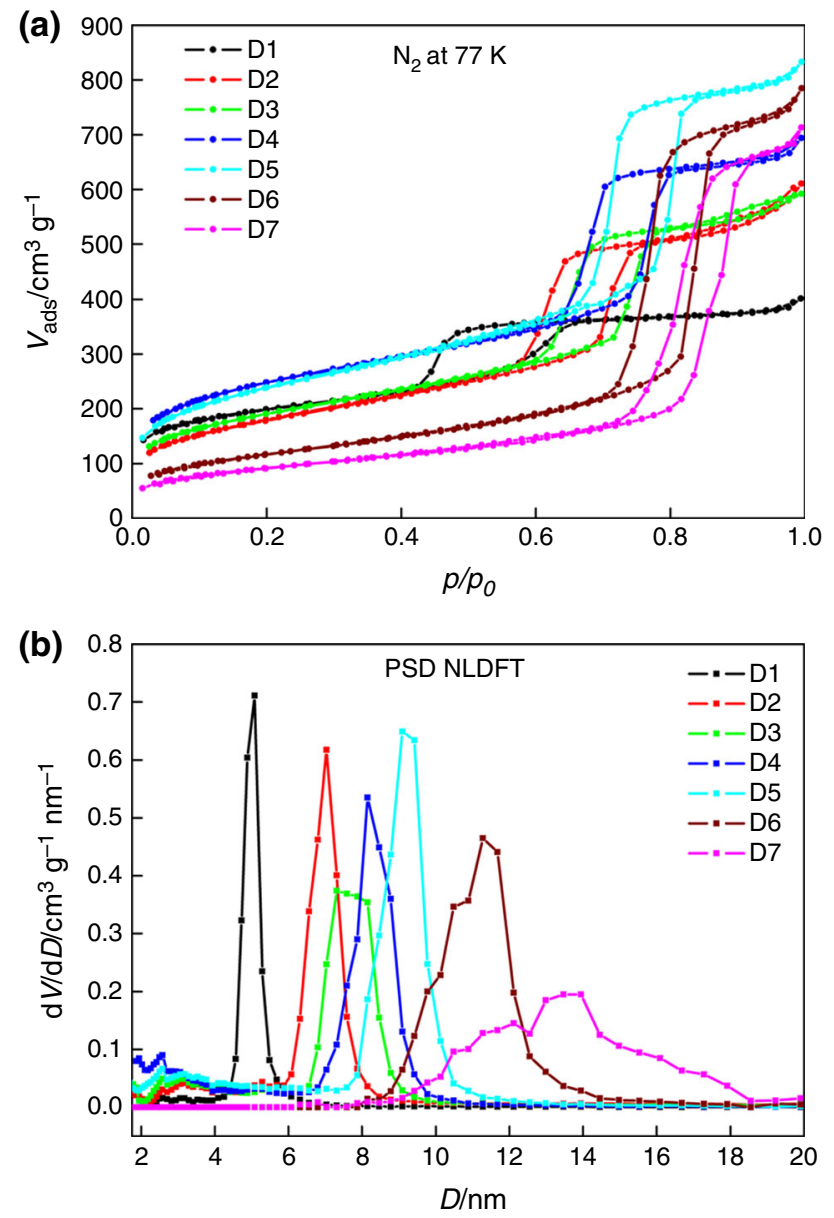

Fig. 2 The low temperature $\mathrm{N}_{2}$ adsorption-desorption isotherms (a) and pore size distribution profiles obtained from NLDFT model (b)
DSC profile of solid-to-liquid phase transition of water confined in one of the studied SBA-15 silicas (D3) is shown in Fig. 3. The first endothermic peaks correspond to the melting of the solid confined in the pores (pore), while the second ones result from the melting of the probe outside the pores (bulk).

In our previous work [22], we reported that experimental protocols may have a serious effect on the position, shape and intensity of the peaks observed in the DSC curve, therefore falsify the TPM results. Thus, in order to define the proper experimental conditions, the role of cooling and heating rates, activation temperature and liquid-to-solid mass ratio was investigated.

The protocols for the DSC measurements that could be found in references vary depending on the studied materials, probes and laboratories. The most common approach involves first quenching the sample with the liquid probe to far below the freezing temperature to avoid the supercooling effect and then the TPM measurements in a heating or in loop mode. The latter method involves heating through the pore melt region until melting of the excess phase just commences (first melting) and then cooling at the same slow rate through the freezing of the dispersed phase and heating again (second melting). In this approach, the pores are open to the exterior; therefore, the presence of the frozen external phase provides nucleation centres for crystallization of the confined liquids once the local equilibrium transition temperature is reached [24]. Frozen sample must be heated with the rate slow enough to allow the continuous thermal equilibrium inside the DSC cell. On the other hand, when the scanning rate is too small, the signal-to-noise effect is large and might disturb the results. In this study, two heating rates were checked: $\beta=1{ }^{\circ} \mathrm{C} \min ^{-1}$ and $\beta=2{ }^{\circ} \mathrm{C} \min ^{-1}$ in the simple heating mode and $\beta=2{ }^{\circ} \mathrm{C} \min ^{-1}$ in loop mode. The results for selected samples are presented in Fig. 4a, b, respectively.

The results presented in Fig. 4 show that there is no need of using very small heating rate or loop mode in order to obtain valuable results. The small differences are observed only between areas and positions of peaks illustrating melting of ice while heating the sample D2 and D6 with rate $\beta=1{ }^{\circ} \mathrm{C} \min ^{-1}$ and $\beta=2{ }^{\circ} \mathrm{C} \min ^{-1}$. It is known that melting transition in porous materials is generally not sharp, but it is spread over a wide temperature range not only due to the distribution of pore size but also because of the fact that various parts of the sample experience temperature increase at slightly different time. The higher the scanning rate and the bigger the amount of sample, the higher the temperature gradient, and the difference in peak intensity and position was observed for D2 and D6. In case of D1, the amount of liquid confined in the pores is very small and all molecules react on the 
Table 1 Characterization parameters for studied materials measured by nitrogen sorption and XRD data

\begin{tabular}{|c|c|c|c|c|c|c|c|c|}
\hline \multirow[t]{3}{*}{ Sample } & \multicolumn{7}{|c|}{ Nitrogen sorption } & \multirow{3}{*}{$\begin{array}{l}\text { XRD } \\
d_{100} / \mathrm{nm}\end{array}$} \\
\hline & \multirow[t]{2}{*}{$V_{\mathrm{mic}} / \mathrm{cm}^{3} \mathrm{~g}^{-1}$} & \multicolumn{3}{|l|}{ NLDFT } & \multicolumn{3}{|l|}{$\mathrm{BJH}$} & \\
\hline & & $S / \mathrm{m}^{2} \mathrm{~g}^{-1}$ & $V / \mathrm{cm}^{3} \mathrm{~g}^{-1}$ & $D / \mathrm{nm}$ & $S / \mathrm{m}^{2} \mathrm{~g}^{-1}$ & $V / \mathrm{cm}^{3} \mathrm{~g}^{-1}$ & $D / \mathrm{nm}$ & \\
\hline D1 & 0.065 & 787 & 0.58 & 5.1 & 600 & 0.53 & 3.5 & 8.8 \\
\hline D2 & 0.005 & 575 & 0.88 & 7.0 & 409 & 0.87 & 5.5 & 9.3 \\
\hline D3 & 0.024 & 639 & 0.88 & 7.3 & 486 & 0.80 & 6.0 & 9.6 \\
\hline D4 & 0.048 & 876 & 1.01 & 8.1 & 858 & 1.01 & 6.5 & 10.5 \\
\hline D5 & 0.005 & 781 & 1.22 & 9.1 & 606 & 1.15 & 7.4 & 11.1 \\
\hline D6 & 0.000 & 396 & 1.14 & 11.3 & 508 & 1.27 & 9.5 & 11.3 \\
\hline D7 & 0.000 & 304 & 1.11 & 13.5 & 374 & 1.14 & 11.3 & 11.3 \\
\hline
\end{tabular}

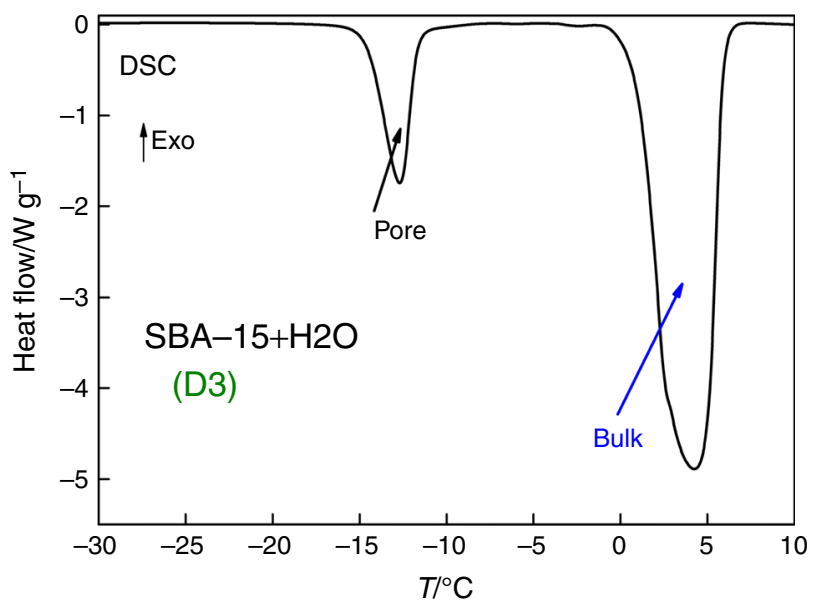

Fig. 3 DSC profile of solid-to-liquid phase transition of water confined in SBA-15 (D3)

temperature changes at the same time. This observation leads to the conclusion that $\beta=1{ }^{\circ} \mathrm{C} \min ^{-1}$ is better for TPM experiments than $\beta=2{ }^{\circ} \mathrm{C} \mathrm{min}{ }^{-1}$. However, since the differences between both results are not very significant and using $\beta=2{ }^{\circ} \mathrm{C} \mathrm{min}^{-1}$ vitally decrease time of measurement, it is recommended for the TPM experiments after calibration procedure. Additionally, from the Fig. 4b, one can conclude that since repeating freezing and melting cycles do not cause any changes in the DSC profiles, all silica samples are not corroded by water and freezing of water inside the pores does not destroy the pore walls or sample structure.

In order to check the role of the speed with which liquid probe solidifies, the effect of different cooling rates prior to the TPM experiment was investigated $\left(\beta=2{ }^{\circ} \mathrm{C} \min ^{-1}\right.$ or $\beta=10^{\circ} \mathrm{C} \min ^{-1}$ ). The DSC profiles (Fig. 5) illustrating the melting of ice inside the SBA- 15 pores are practically the same for all studied samples independently on cooling
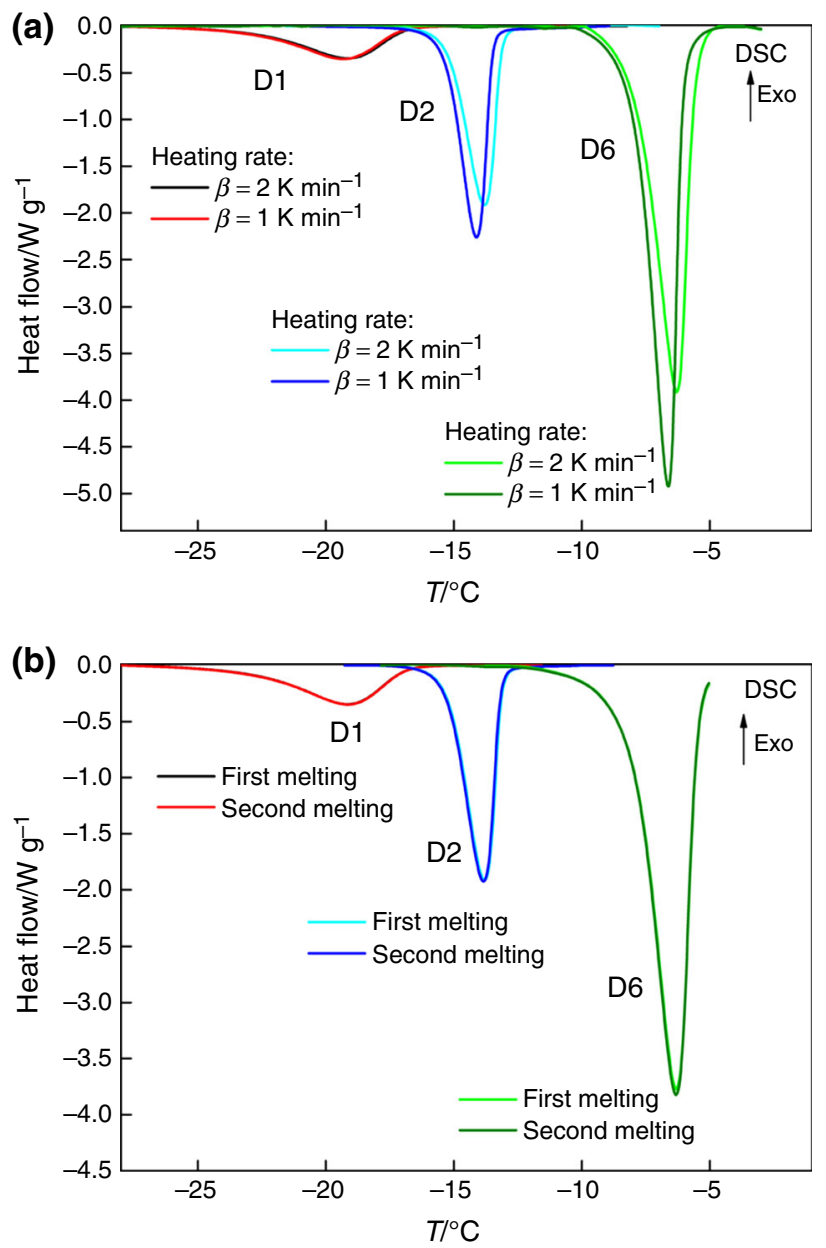

Fig. 4 DSC profiles obtained during heating the samples with different heating rate (a) or with use loop mode (b)

speed. Freezing the sample with high cooling rate allows decrease time needed for the experiments and thus is recommended for TPM protocol. 


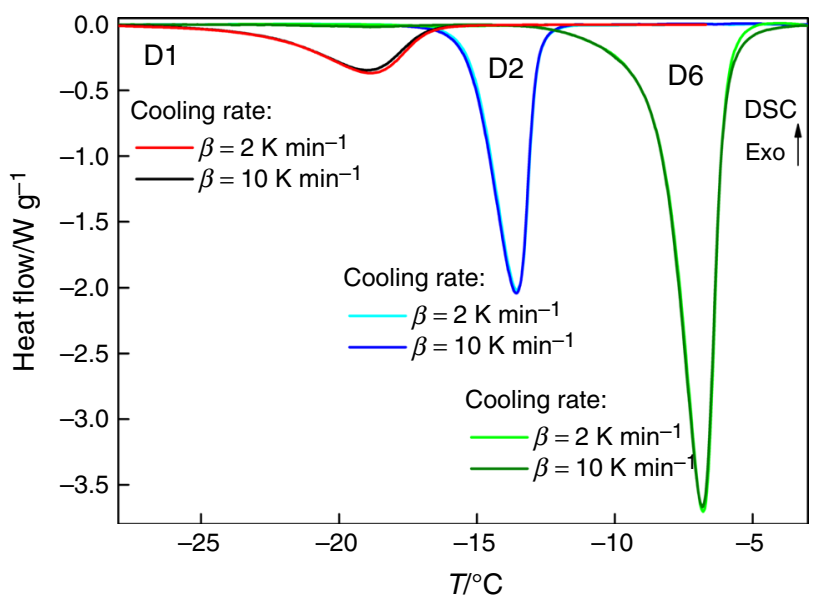

Fig. 5 Part of DSC profiles illustrating the melting of ice inside the SBA-15 pores after cooling the sample with different rate

Another important question is how the interaction of pore wall impacts the water TPM results. SBA-15 is reported to be rather hydrophobic even it shows relatively high water sorption capacity (for $\mathrm{D}=5 \mathrm{~nm}$, it is $0.84 \mathrm{~g} \mathrm{~g}^{-1}$ ) [30]. It is known that the mesoporous materials have abundant number of silanol groups because of the amorphous surface structure; therefore, a large amount of water can be sorbed on them followed by capillary condensation [31]. It is known from the work of Pantalei et al. [32] that it is possible to separate the contribution of the protons in the water-mesoporous silica system and thus to determine of the arrangement of water molecules in respect to silanols groups. It is based on their observation that the hydrogen bond created between the water proton and the oxygen of the silanol group is much stronger than the hydrogen bonds present between molecules of bulk water.

Pioneering studies of water sorption on surface of silica have shown that the molar heats of adsorption calculated for the stepwise adsorption of water vapour are equal to 104.6, 66.9 and $39.7 \mathrm{~kJ} \mathrm{~mol}^{-1}$ for first, second and third water layer, respectively [33, 34]. The authors suggested that in the case of the first water layer formation, the water molecules were in a more localized state thus the calculated value of isosteric heat and the differential entropy was higher than for second and third layer. More recent works $[35,36]$, extended on high silica zeolites and mesoporous silicas (BEA, SBA-15, MCM-41), maintained earlier conclusions demonstrating that the measured heat of adsorption of water $\left(100<q_{\text {diff }}<44 \mathrm{~kJ} \mathrm{~mol}^{-1}\right)$, directly related to the bond energies between water and sorption centres, was larger than the heat of liquefaction of water $\left(44 \mathrm{~kJ} \mathrm{~mol}^{-1}\right)$ in the whole coverage. The type of silanol groups, being the sorption centres, as well as the geometry of water adsorptive adduct, was also considered as factors affecting the sorption process and measured heats of adsorption. Their role turned out to be more decisive.
The surface silanol groups of mesoporous materials are three types, and each of them is supposed to form different interaction with water molecules thus influences the TPM outcomes. Cauvel et al. [37] observed that the nature and local arrangement of surface groups in mesoporous silica (MCM-41) can cause either its hydrophobic or hydrophilic behaviour.

Several kinds of silanol groups can be distinguished in the IR spectrum of mesoporous silicas. The freely osculating isolated $(\mathrm{SiO})_{3} \mathrm{Si}-\mathrm{OH}$ are characterized by the band at $3745 \mathrm{~cm}^{-1}$, while mutually hydrogen-bonded species give the band at lower frequencies. The $3720 \mathrm{~cm}^{-1}$ band is usually assigned to vicinal silanols $(\mathrm{SiO})_{3} \mathrm{Si}-\mathrm{OH}-\mathrm{OH}-$ $\mathrm{Si}(\mathrm{SiO})_{3}$, while the broad band at $3550 \mathrm{~cm}^{-1}$ is typical of linked silanols. According to Cauvel, isolated silanols weakly interact with water, while vicinal and linked spaces nearly freely interact. The occurrence of H-bonding renders the vicinal silanols more acidic, what stays in agreement with calculations on model system [38]. The authors concluded that isolated silanols are not prone to engage in $\mathrm{H}$-bonding with water and the adsorption takes place on species already mutually interacting.

The IR spectra of selected studied SBA-15 materials in the region of hydroxyl band vibration are presented in Fig. 6.

Depending on the mesopore diameter, the studied materials exhibited the various populations of different type of silanol groups (Fig. 6a). In all samples, the mesopore surface is populated in majority with the isolated silanols $(\mathrm{SiO})_{3} \mathrm{Si}-\mathrm{OH}$ groups $\left(3745 \mathrm{~cm}^{-1}\right)$. The abundance of hydrogen-bonded silanols (3720, 3680 and $3550 \mathrm{~cm}^{-1}$ bands) is the highest in D1 and noticeably decreases with increasing of the pore size. It stays in agreement with the statement that narrower mesopores of silica facilitate formation of hydrogen-bonded silanols groups [39]. High temperature treatment $\left(30 \mathrm{~min}\right.$ in $650{ }^{\circ} \mathrm{C}$ ) applied to studied samples led to the distinctive reduction of amount of highly linked silanols, while those being isolated remained untouched (grey line in Fig. 6a).

Sorption of water on studied samples, independently on their pore diameter thus different population of isolated, vicinal and hydrogen-bonded silanols, resulted in perturbation of the isolated and vicinal silanols by H-bonding interactions with water molecules. Figure $6 \mathrm{~b}$ shows the difference IR spectra in which negative bands at 3745 and $3720 \mathrm{~cm}^{-1}$ and the simultaneous evolution of a shifted, broadened and intensive band at ca. $3350 \mathrm{~cm}^{-1}$ $\left(\Delta v=400 \mathrm{~cm}^{-1}\right)$ were observed. In conclusion, majority of the vicinal silanols are bonded primarily with water. Such conclusion can be derived from the comparison of the normalized to the same high negative IR bands of silanols in the spectra recorded upon the sorption of the first dose of water (red lines) and the dose of water sufficient to cover 

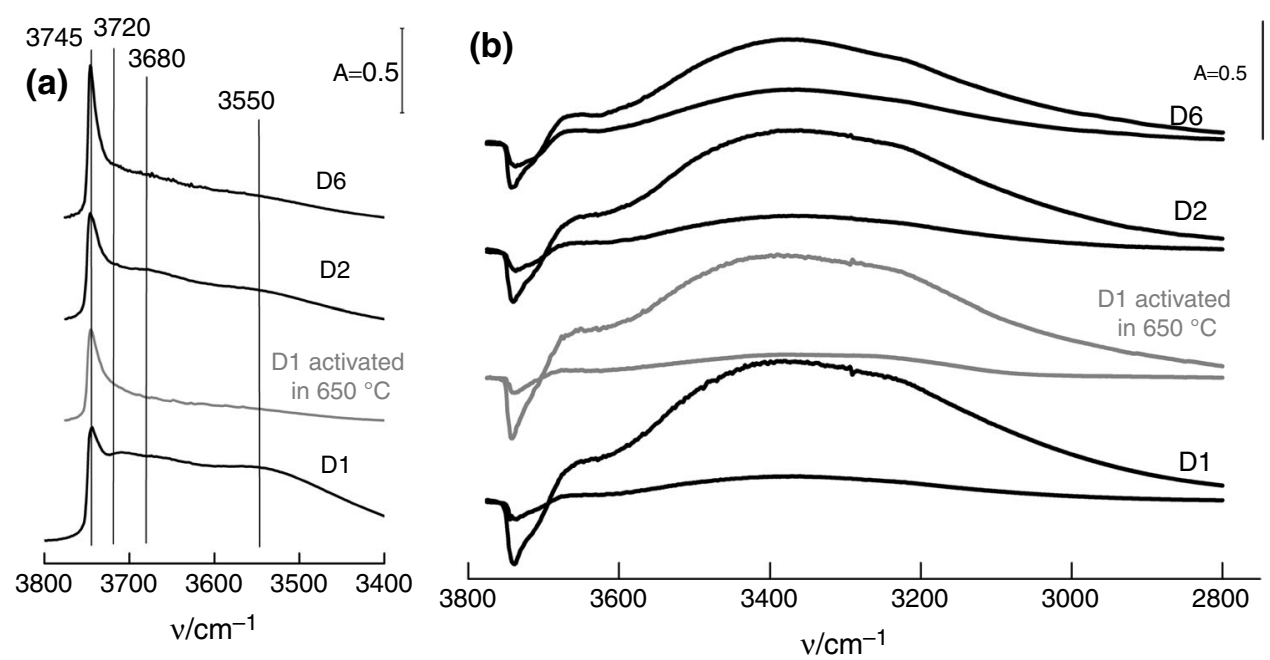

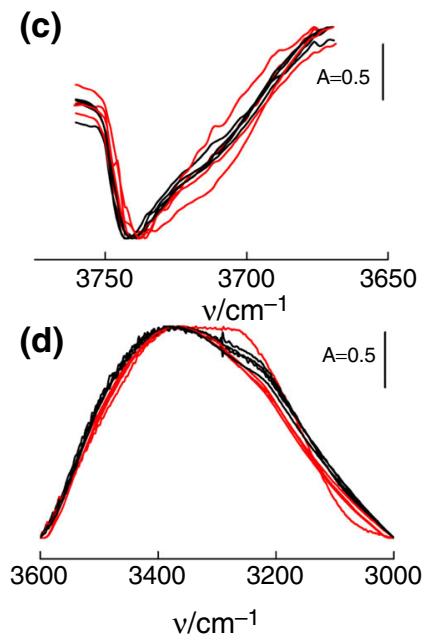

Fig. 6 IR spectra of silanol groups in SBA-15 materials of various pore diameters (a), difference IR spectra of silanol groups after sorption of water (b), hydrogen-bonded silanol groups shifted to lower frequencies being normalized to the same high (c), negative bands of silanol groups normalized to the same high, for a series of studied samples $(\mathbf{d})$ all surface silanols (black lines in Fig. 6c). These spectra exhibited the slight different proportion between the $3745 \mathrm{~cm}^{-1}$ (isolated $\mathrm{Si}-\mathrm{OH}$ ) and $3720 \mathrm{~cm}^{-1}$ (vicinal silanols) bands confirming that vicinal silanols react at first upon sorption of water. However, for different samples, it has been noticed that, despite various populations of different type of silanol groups in those samples, the normalized spectra for all of them demonstrated the same characteristic. It means that there is no difference in the interaction of water with the silanol group of both types. This conclusion can be supported by the observation that the width at half maximum of $3600-3000 \mathrm{~cm}^{-1}$ of the $\mathrm{O}-\mathrm{H}$ stretching band (Fig. 6d) is of the same value, independently neither on consecutive portions of water nor from type of sample, suggesting that the band originated from $\mathrm{H}$-bonding is associated with $\mathrm{OH}$ groups manifesting an intrinsic homogeneity of silanol groups. This phenomenon can be explained by the basicity of water molecule being high enough to not provide the possibility of discrimination between sites of the same quality having small differences in acid strength.

Worth underlining is that the probability of forming the H-bond involving more linked silanol, represented by a complex downshifted band at $3550 \mathrm{~cm}^{-1}$, cannot be detected in IR spectra. The overlapping of the H-bond component present in mesoporous silicas, especially in D1, with the H-bond bands formed after water adsorption did not allowed for identification of the mentioned above effect.

Understanding the nature of silanol species is important in helping to study the influence of temperature activation on TPM results. The most wanted situation is when there is no need for any activation procedure before DSC measurements because the water TPM method is especially devoted for study the porosity of samples that can change their properties in drying process. However, in order to check the practical impact of temperature pre-treatment on TPM results, three additional type of experiments were taken: 1) the TPM measurement was made on the sample firstly calcinated in $400{ }^{\circ} \mathrm{C}$ for $30 \mathrm{~min}, 2$ ) before TPM experiments the sample was activated in $650{ }^{\circ} \mathrm{C}$ for 30 min, and 3) the TPM was done on the sample without any previous treatment. The obtained results are shown in the Fig. 7.

The most visible differences are observed in case of the sample with the smallest pore diameter (D1), while in silica with the largest pores (D6), the activation temperature has no influence on TPM results. It is also seen in the Fig. 7 that even for D1, the DSC profiles obtained after activation of the silica in $400{ }^{\circ} \mathrm{C}$ are the same as received without any pre-treatment. It suggests that decline in number of vicinal and highly linked silanols results in changes in the amount of water that undergoes solidification inside the SBA-15 channels. This statement is supported by the fact, reported in our previous paper [39], that the activation in $400{ }^{\circ} \mathrm{C}$ does not decrease hydrogen-bonded silanols groups presented in SBA-15. In order to explain the obtained results, the presence of non-freezing water inside the silica channels should be considered. The existence of boundary layer of non-freezable water at silica pore walls is a common knowledge. It is also known that the arrangement and mobility of molecules in this layer is affected by $\mathrm{SiOH}$ groups at the pore walls [40]. During heating silica up to the temperature higher than $550{ }^{\circ} \mathrm{C}$, condensation of linked silanol groups takes place and siloxane bridges $(\mathrm{Si}-\mathrm{O}-\mathrm{Si})$ are formed [31]. After this activation, water can be 

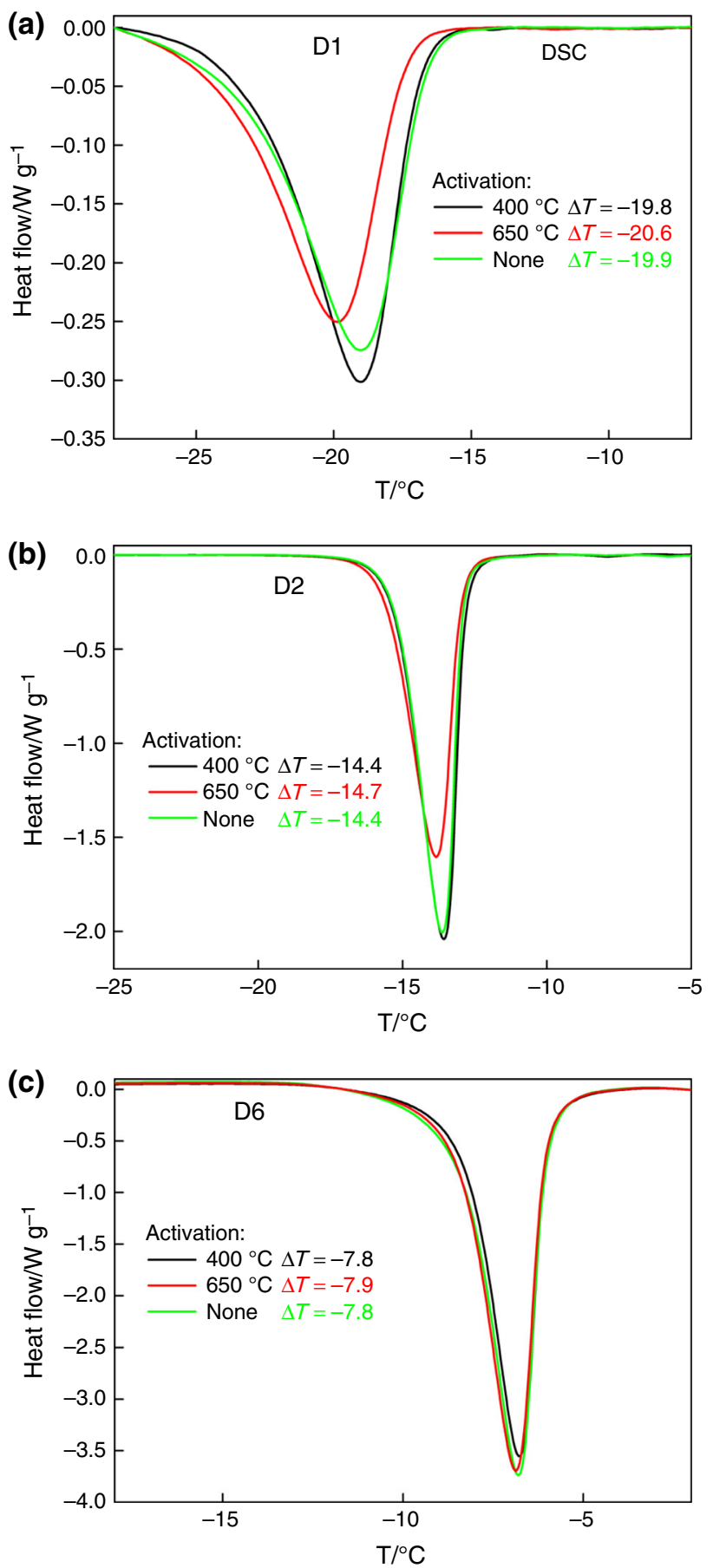

Fig. 7 DSC profiles illustrating the melting of ice inside the D1 (a), D2 (b), and D6 (c) silica pores after sample pretreatment in various temperatures

physisorbed on the nearly dehydroxilated surface; however, some dissociation of water occurs with the corresponding generation of new adsorption sites by hydrolysis of siloxane bridges [37]. This modification of the surface can be responsible for differences observed between samples D1 and the others. The layer of non-freezing water is bigger when the water particles are chemisorbed on adsorption sites origin from siloxane bridges. The population of them is the highest in D1, which results in less intensity and smaller area of DSC peak reflecting the melting of ice inside that silica after the activation at $650{ }^{\circ} \mathrm{C}$, in comparison with other samples. The amount of non-freezing water calculated as a difference between the total liquid content and the freezing water obtained from melting endotherms confirms this statement: It was equal to $0.42,0.43$ and $0.50 \mathrm{gg}^{-1}$ for sample activated in $400{ }^{\circ} \mathrm{C}$, no activated sample and activated in $650{ }^{\circ} \mathrm{C}$, respectively.

The results presented above lead to the conclusion that sample thermal treatment undertaken before water TPM experiments is not only unnecessary but also can lead to erroneous results and thus is unadvisable.

One more experimental factor that certainly should not be ignored is the relative mass of solid silica and water taken for the TPM measurements. In our previous work [22], we observed that liquid-to-solid mass ratio $(\mathrm{l} / \mathrm{m})$ significantly influenced the n-alkane TPM results. In case of water TPM, this factor was investigated by a few authors but only with partially filled pores. Schreiber et al. [27] or Findenegg et al. [40] observed that whereas melting scans exhibited only a single peak, two or three peaks appeared in the freezing scans, depending of the degree of pore filling. They attributed this behaviour to the existence of two or more distinct arrangements of solid and liquid in the pores. In our investigation, we focused on study the behaviour of water inside the pores which are completely filled but with different solid-to-liquid mass ratio. Obtained results for selected silicas are plotted in the Fig. 8 .

Based on the values of pore volume derived from NLDFT calculations (Table 1), one can notice that in all cases presented in Fig. 8, silica pores were expected to be totally filled. However, the dependence of melting profiles on $l / m$ is clearly seen. For silicas with the smallest pore size (D1 and D2), melting peak position is almost independent on the $l / m$ ratio but peak area decreases with increasing liquid mass. The exceptions are the lowest values of $l / m$, for which the area under the peaks is the smallest. Probably, in these cases, pores are not totally filled, even if the amount of water was sufficient, due to the lowest sorption capacity of the silica with the smallest pore size. The variation of pore area with $l / m$ suggests that the layer of non-freezing water is not stable but varies not only with the temperature [40] but also with $\mathrm{l} / \mathrm{m}$ and is the biggest for the highest water to silica mass ratio. Indeed, the amounts of non-freezing water calculated for all the samples at different moisture contents and collected in Table 2 confirm this supposition.

For silica D1, the lowest amount of non-freezing water was observed when the liquid-to-solid mass ratio was about 2. For higher $l / m$, the $t$ values are bigger. However, in such small pores, the differences in $t$ are not really significant. 

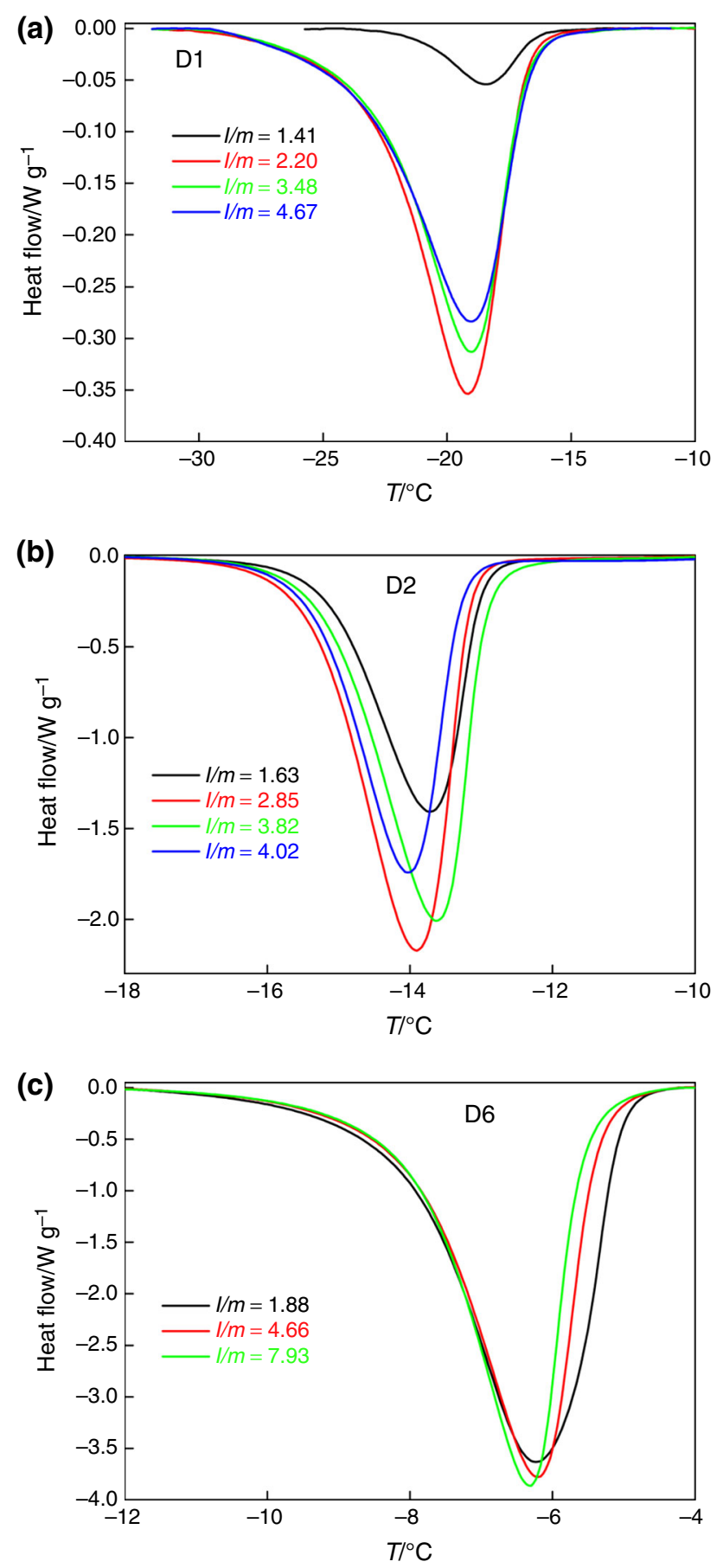

Fig. 8 DSC profiles illustrating the melting of ice inside the D1 (a), D2 (b) and D6 (c) silica pores depending on solid-to-liquid mass ratio $(\mathrm{l} / \mathrm{m})$

The bigger amount of non-freezing water is observed for the silicas with wider pore diameter. In case of D2, the amount of water which does not undergo solidification clearly influences the DSC outcome. The smaller the $l / m$, the higher the intensity of the pore melting peak is. In case of D6, the amount of non-freezing water seems not to affect the DSC profile seriously: peak areas and positions are almost the same for all studied moisture contents.

The observation described above can probably be explained by taking into account the differences in the condition in which the freezing occurs. When the amount of excess liquid water is large, the majority of pores are fully covered by it and the liquid inside the pores does not experience gas atmosphere. Since the density of liquid water is higher than density of water in solid state, the ice created during solidification exerts higher pressure on the liquid layer present next to the pore walls comparing to the situation of smaller $\mathrm{l} / \mathrm{m}$ when the bigger population of pores remains open. In the latter case, the phase transition from liquid-to-solid state undergoes under normal pressure. It is known that pore filling in SBA-15 proceeds in layerby-layer mode [40]. Higher pressure exerted on the layers makes them "ticker" that lead to the situation that a bigger population of water layer experiences the interaction with silanol groups from pore walls. As it was said before, the hydrogen bond created between the water proton and the oxygen of the silanol group is much stronger than the hydrogen bonds present between molecules of water [3236]. It means that the more water layers interact with silanols, the bigger the thickness of the non-freezing liquid layer is. It is well seen for the sample D2 (Table 2) where the number of non-freezing water monolayers increases with liquid-to-solid mass ratio.

All the investigation presented above lead to the conclusion that in order to obtain valuable results from water TPM, the following experimental protocol should be used: Certain amount of the sample should be put into weighted DSC pan without any previous thermal treatment, then water should be added and the pan needs to be sealed. The amounts of solid and liquid must be carefully controlled in order to reach their mass ratio about 3 . Then, the sample should be quenched far below the equilibrium freezing ( $-60{ }^{\circ} \mathrm{C}$ is enough) with cooling rate $\beta=10{ }^{\circ} \mathrm{C} \min ^{-1}$ and heated up to the room temperature with $\beta=2{ }^{\circ} \mathrm{C} \min ^{-1}$. After the TMP experiments, a small hole should be made in the crucible's lid and the sample needs to be heated in order to evaporate the liquid component and determine the sample mass.

To transform the obtained TPM profile into the pore size distribution (PSD), the temperature axis must be converted into a pore size scale and the heat flow output into a differential pore volume. The basis for relating temperature to pore radius is through the Gibbs-Thomson equation or the empirical one. The detailed procedure of this conversion was described earlier [21, 22, 24, 39]. Generally, after a baseline subtraction step that effectively removes the underlying heat capacity contribution to the DSC signal, the heat flow curve, $\mathrm{d} Q / \mathrm{d} t$, is converted to $\mathrm{d} V_{\mathrm{p}} / \mathrm{d} R_{\mathrm{p}}$ according to the equation: 
Table 2 Amount of non-freezing water per gram of silica D1, D2 and D6 at different moisture contents $(t)$, expressed as thickness of nonfreezing liquid layer $(t *)$ and as the amount of monolayers $(w)$

\begin{tabular}{|c|c|c|c|c|c|c|c|c|c|c|c|}
\hline \multicolumn{4}{|l|}{ D1 } & \multicolumn{4}{|l|}{ D2 } & \multicolumn{4}{|l|}{ D6 } \\
\hline$(\mathrm{l} / \mathrm{m})$ & $t / \mathrm{g} \mathrm{g}^{-1}$ & $t^{*} / \mathrm{nm}$ & $w$ & $(\mathrm{l} / \mathrm{m})$ & $t / \mathrm{g} \mathrm{g}^{-1}$ & $t^{*} / \mathrm{nm}$ & $w$ & $(\mathrm{l} / \mathrm{m})$ & $t / \mathrm{g} \mathrm{g}^{-1}$ & $t^{*} / \mathrm{nm}$ & $w$ \\
\hline 1.41 & 0.44 & 0.56 & 2 & 1.63 & 0.61 & 1.06 & 4 & 1.88 & 0.35 & 0.88 & 3 \\
\hline 2.20 & 0.30 & 0.38 & 1 & 2.85 & 0.42 & 0.73 & 2 & 4.66 & 0.48 & 1.21 & 4 \\
\hline 3.46 & 0.42 & 0.53 & 2 & 3.82 & 0.55 & 0.96 & 3 & 7.93 & 0.38 & 0.96 & 3 \\
\hline 4.67 & 0.43 & 0.55 & 2 & 4.02 & 0.61 & 1.06 & 4 & & & & \\
\hline
\end{tabular}

The thickness of non-freezing liquid layer $t^{*}$ was calculated based on the values of the surface area obtained from NLDFT method. The amount of monolayers present in the silica channels was obtained under assumption that the van der Waals radius of water is equal to $0.3 \mathrm{~nm}$ [41]

$\frac{\mathrm{d} V_{\mathrm{p}}}{\mathrm{d} R_{\mathrm{p}}}=\frac{\mathrm{d} Q}{\mathrm{~d} t} \frac{\mathrm{d} t}{\mathrm{~d}(\Delta T)} \frac{\mathrm{d}(\Delta T)}{\mathrm{d} R_{\mathrm{p}}} \frac{1}{m \Delta H_{\mathrm{f} \rho}}$

where $\mathrm{d}(\Delta T) / \mathrm{d} t$ is the scanning rate of the DSC experiment, $m$ the mass of dry porous material, and $\Delta H_{\mathrm{f}}$ and $\rho$ the heat of fusion and density for the probe fluid, respectively. The relation $\mathrm{d}(\Delta T) / \mathrm{d} R_{\mathrm{p}}$ may be given by the Gibbs-Thomson equation but because of unavoidable disregard of the temperature dependence of parameters used in this equation, it may cause systematic deviations in the calculated PSD and lead to erroneous results. In order to avoid this problem, calibration procedure is advice to be implied.

In order to use the solid-to-liquid phase transition water as a thermoporosimetry probe, it is necessary to establish the mathematical relationships between the diameter of the pores $(D)$ and the corresponding phase transition temperature depression $\Delta T$. The overall trend for most thermoporosimetry experiments cited in the references is that of a linear dependence between $\Delta T$ and the inverse of pore radius $1 / R_{\mathrm{p}}$ [24-27]. This assumption allows fitting experimental data to the modified Gibbs-Thomson equation which take into account the presence and thickness of the non-freezing liquid layer $t$ (Eq. 3).

$R_{\mathrm{p}}=\frac{K}{\Delta T}+t$

However, it is also known that there is a subtle nonlinearity in the melting point data that hints of departure from the simplified expression 3. To avoid this problem, Billamboz et al. proposed to use exponential dependence between $R_{\mathrm{p}}$ and $1 / \Delta T$ according to the Eq. (4):

$R_{\mathrm{p}}=t \cdot \exp \left(-\frac{1}{c \cdot \Delta T}\right)$

where $c$ is a parameter depending on the solvent and $t$ is the thickness of the layer that does not undergo solidification [42]. They received reasonable results for serious of organic liquids.

To establish the calibration relationships based on the experiments presented in this paper, the pore diameters
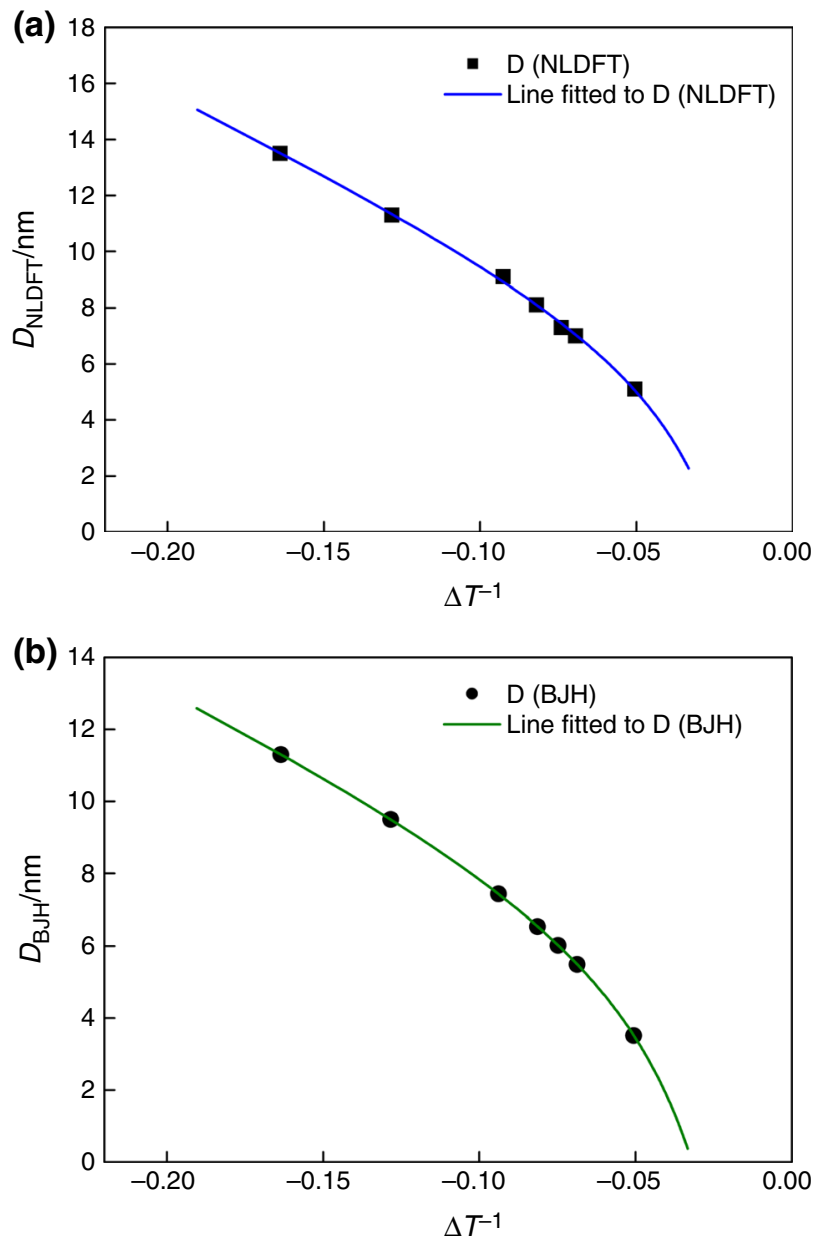

Fig. 9 Fitting relations between the pore diameters (D) obtained from NLDFT (a) and BJH calculations (b) and the inverse of melting point depression of water with nonlinear functions

previously determined from the nitrogen sorption (NLDFT and $\mathrm{BJH}$ ) method were plotted versus the inverse of the melting point depression and fitted with empirical functions (Fig. 9a, b, respectively). We decided to use both NLDFT and BJH method because even NLDFT is recommended for cylindrical pore analysis, the results of pore diameter value 

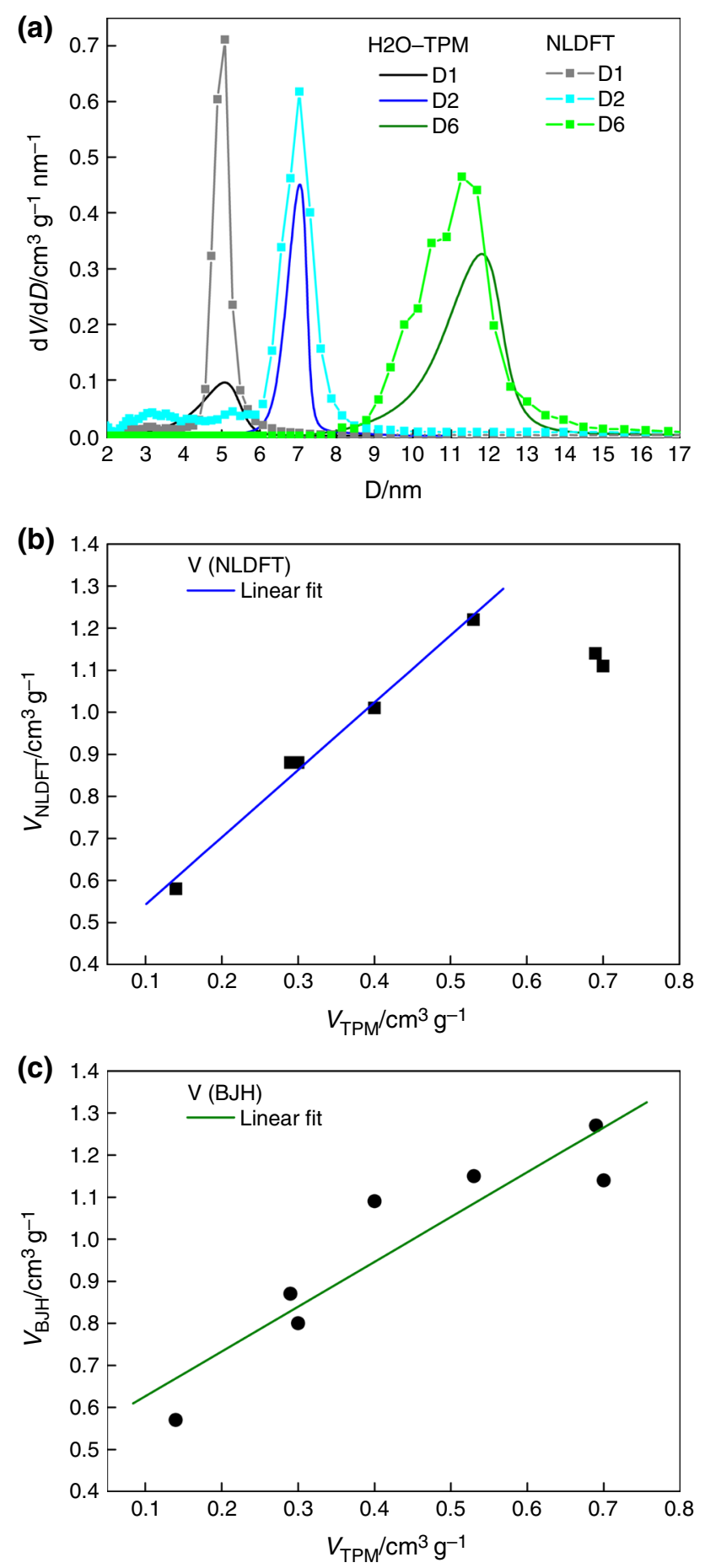

Fig. 10 Pore size distribution obtained from water TPM with use empirical equations based on NLDFT calculations (a), relations between the pore volumes $(\mathrm{V})$ obtained from water TPM and NLDFT (b) or BJH (c) methods

$(D)$ obtained for SBA-15 with the biggest pore size seem to be overestimated (Table 1). It is well seen in the case of D7 for which pore diameter derived from NLDFT is higher than that obtained from XRD patterns.
Table 3 Parameters of the empirical functions obtained by their fitting to the experimental data (cf. Figs. 9, 10). $R^{2}$ denotes values of the correlation coefficient between the experimental and fitted data

\begin{tabular}{|c|c|c|c|c|}
\hline & \multicolumn{2}{|l|}{$D / \mathrm{nm}$} & \multicolumn{2}{|c|}{$V / \mathrm{cm}^{3} \mathrm{~g}^{-1}$} \\
\hline & NLDFT & $\mathrm{BJH}$ & NLDFT & BJH \\
\hline$a$ & -52.25 & -40.00 & 1.60 & 1.07 \\
\hline$b$ & 6.07 & 6.23 & 0.38 & 0.52 \\
\hline$c$ & 0.18 & 0.24 & - & - \\
\hline$R^{2}$ & 0.999 & 1 & 0.989 & 0.852 \\
\hline
\end{tabular}

Analysis of the experimental data showed that the obtained relation $D(1 / \Delta T)$ was neither linear nor exponential thus a simple nonlinear function (Eq. 5), containing three adjustable parameters $\mathrm{a}, \mathrm{b}$ and $\mathrm{c}$, quantifying inverse proportional, constant and proportional contribution of $\Delta T$, was fitted.

$y=\frac{a}{\Delta T}+b+c \cdot \Delta T$

It is important to emphasize that this approach is not consistent from a physical point of view when $\Delta T$ tends towards infinity. In this case, $D$ tends towards infinity as well, whereas it should converge towards a constant value as it is for linear or exponential relations. Moreover, it does not give any information about the thickness of nonfreezing layer since it is not derived from Gibbs-Thomson equation. However, we decided to use this approach anyway, because it works very well in the practical range of $\Delta T$ relevant for TPM analysis and gives very good fitting results. Least-square fitting was performed using the Solver optimization procedure (from Excel/MS Office 10). In Table 2, all the fitting parameters were listed.

The use of empirical equations allows obtaining pore size distribution (PSD) profiles out of the DSC curves. In order to calculate the values of pore volume, the following density of ice was used: $\rho=0.917 \cdot\left(1.032-1.17 \cdot 10^{-4} \mathrm{~T}\right)$ $\mathrm{gcm}^{-3}[24]$.

As it can be seen in Fig. 10a, the areas under the TPMPSD peaks, that represent the pore volume, remain much smaller than the corresponding pore volumes obtained using nitrogen adsorption methods. The reason is not entirely clear. Probably, the TPM pore volume is underestimated because part of the absorbed solvent does not undergo solidification. However, the calculated amount of non-freezing water is not big and should not have a dramatic effect on the pore volume. On the other hand, it is worth to notice that the pore volume determined from NLDFT calculations is overestimated especially in case of silicas with larger pore diameter. Certainly, in order to explain observed phenomenon, further investigations are needed. 

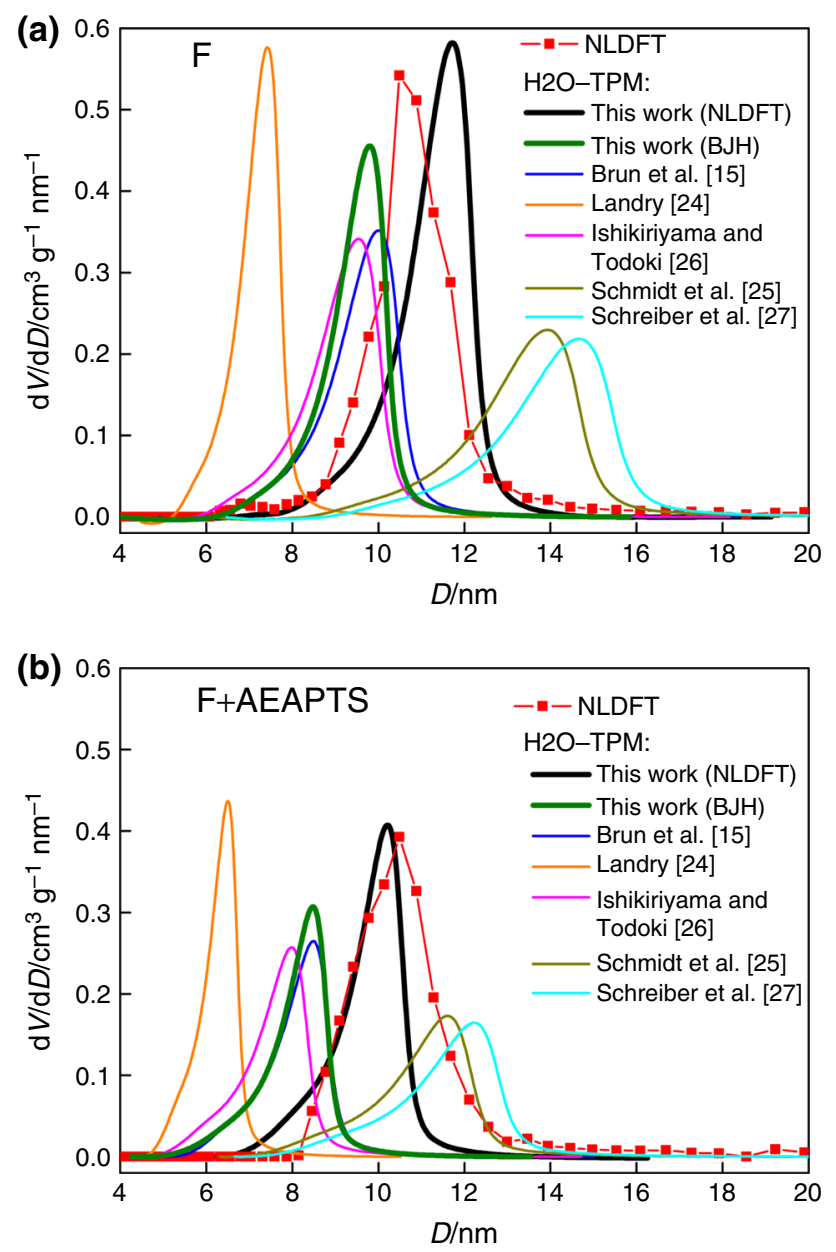

Fig. 11 PSD profiles derived from NLDFT and water TPM with use different empirical equations for SBA-15 before (a) and after (b) functionalization procedure

In order to establish the calibration equations for pore volumes, the values of pore volume derived from TPM were plotted versus the corresponding NLDTF and BJH results (Fig. 10 b, c, respectively). The linear relationship between the water TPM and NLDFT derived pore volume was found for the silicas with the pore size below $10 \mathrm{~nm}$ (D1-D5). The parameters of the fitted linear equations $(y=a x+b)$ were listed in Table 3 . The pore volumes of the silicas containing pores larger than $10 \mathrm{~nm}$ do not follow that linear trend suggesting that water is less efficient in filling of such pores or the NLDFT gave false results. BJH derived relationship is poorly linear confirming that NLDFT better describes porosity of cylindrical mesoporous silica.

Experimental protocol and calibration equations were use to study the porous properties of SBA-15 silica that was not use before (denoted as F) and the same material after functionalization with AEAPTS (named F-AEAPTS). Functionalization procedure may change not only the chemical properties of studied silica but also the porous parameters of the material-pore diameter and volume. In the Fig. 11, the results obtained for F1 and for F+AEAPTS with use NLDFT and water thermoporosimetry were plotted. Additionally, the PSD profiles calculated based on the equations provided by other authors were placed for comparison.

It can be seen from Fig. 11 that PSD profiles regardless of the method used for measurements or type of equation allow to observe the differences in porous properties between SBA-15 silica before and after functionalization procedure. The values of pore diameter and volume, collected in Table 4, lead additionally to the conclusion that the equations proposed in this work give the results much closer to the values derived from liquid nitrogen experiments than the equations proposed by other authors. It is seen especially in case of pore volume. However, they also confirm that for silicas with pore diameter bigger than $10 \mathrm{~nm}$, the differences between water thermoporosimetry and nitrogen sorption outcomes become higher.

Table 4 Pore diameter and pore volume values of SBA-15 (F) sample before and after functionalization with AEAPTS (F+), derived from nitrogen adsorption $\left(\mathrm{N}_{2}\right)$ and TPM experiments with used different equation for calculations

\begin{tabular}{|c|c|c|c|c|c|c|c|c|c|}
\hline & \multicolumn{2}{|c|}{$\begin{array}{l}\text { Nitrogen } \\
\text { sorption }\end{array}$} & \multicolumn{7}{|c|}{ Water thermoporosimetry } \\
\hline & $\begin{array}{l}\text { NL- } \\
\text { DFT }\end{array}$ & $\mathrm{BJH}$ & $\begin{array}{l}\text { This work } \\
\text { NLDFT }\end{array}$ & $\begin{array}{l}\text { This work } \\
\text { BJH }\end{array}$ & $\begin{array}{l}\text { Brun et al. } \\
{[15]}\end{array}$ & $\begin{array}{l}\text { Landry } \\
{[24]}\end{array}$ & $\begin{array}{l}\text { Ishikiriyama and } \\
\text { Todoki [26] }\end{array}$ & $\begin{array}{l}\text { Schreiber et al. } \\
\text { [27] }\end{array}$ & $\begin{array}{l}\text { Schmidt et al. } \\
{[25]}\end{array}$ \\
\hline \multicolumn{10}{|l|}{$\mathrm{F}$} \\
\hline$D / \mathrm{nm}$ & 10.5 & 8.6 & 11.7 & 9.8 & 10.0 & 7.4 & 9.5 & 14.6 & 13.9 \\
\hline $\begin{array}{l}V / \\
\mathrm{cm}^{3} \mathrm{~g}^{-1}\end{array}$ & 1.11 & 1.20 & 1.05 & 0.70 & 0.66 & 0.66 & 0.66 & 0.66 & 0.66 \\
\hline \multicolumn{10}{|l|}{$\mathrm{F}+$} \\
\hline$D / \mathrm{nm}$ & 9.8 & 8.0 & 10.2 & 8.5 & 8.5 & 6.5 & 8.0 & 12.2 & 11.6 \\
\hline $\begin{array}{l}V / \\
\mathrm{cm}^{3} \mathrm{~g}^{-1}\end{array}$ & 0.75 & 0.85 & 0.64 & 0.44 & 0.41 & 0.41 & 0.41 & 0.41 & 0.41 \\
\hline
\end{tabular}




\section{Conclusions}

The results presented in this study demonstrate that the behaviour of water confined inside the ordered mesoporous SBA-15 silica is under influence of factors that should be taken into account in order to obtain reliable TPM outcomes. The most important is the solid-to-liquid mass ratio that implies the amount of pores that are fully covered by liquid and thus influence the thickness of non-freezing liquid layer. The heating and cooling rate as well as the previous thermal treatment turned out not to impact the TPM results neither. These observations allowed finding the simply experimental protocol that give a reliable pore characteristic within $30 \mathrm{~min}$. Based on the refined methodology, a calibration procedure was undergone and experimental equations were established. We believe that this work will contribute to making the thermoporosimetry method more popular and more useful for wider spectrum of potential users.

Acknowledgements The research project was financially supported by Grant No. 2012/07/B/ST5/00911 from the National Science Centre, Poland. IR part of this work was financed by Grant No. 2013/09/ B/ST5/00066 from the National Science Centre, Poland. The part of the measurements was carried out with the equipment purchased thanks to the financial support of the European Regional Development Fund in the framework of the Polish Innovation Economy Operational Program (Contract No. POIG.02.01.00-12-023/08).

Open Access This article is distributed under the terms of the Creative Commons Attribution 4.0 International License (http://crea tivecommons.org/licenses/by/4.0/), which permits unrestricted use, distribution, and reproduction in any medium, provided you give appropriate credit to the original author(s) and the source, provide a link to the Creative Commons license, and indicate if changes were made.

\section{References}

1. Taguchi A, Schuth F. Ordered mesoporous materials in catalysis. Microporous Mesoporous Mater. 2005;77:1-45.

2. Davis ME. Ordered porous materials for emerging applications. Nature. 2002;417:813-21.

3. Riikonen J, Salonen J, Lehto V-P. Utilising thermoporometry to obtain new insights into nanostructured materials. J Therm Anal Calorim. 2011;105(3):823-30.

4. Salonen J, Kaukonen A, Hirvonen J, Lehto V-P. Mesoporous silicon in drug delivery applications. J Pharm Sci. 2008;97:632-53.

5. Riikonen J, Makila E, Salonen J, Lehto V-P. Determination of the physical state of drug molecules in mesoporous silicon with different surface chemistries. Langmuir. 2009;25(11):6137-42.

6. Jane A, Dronov A, Hodges R, Voelcker A. N.H. Porous silicon biosensors on the advance. Trends Biotechnol. 2009;27:230-9.

7. Iza M, Woerly S, Danumah C, Kaliaguine S, Bousmina M. Determination of pore size distribution for mesoporous materials and polymeric gels by means of DSC measurements: thermoporometry. Polymer. 2000;41:5885-93.

8. Johnston GP, Smith DM, Melendez I, Hurd AJ. Compression effects in mercury porosimetry. Powder Technol. 1990;61:289-94.
9. Wastermark S, Use of mercury porosimetry and nitrogen adsorption in characterisation of the pore structure of mannitol and microcrystalline cellulose powders, granules and tablets. Academic Dissertation, Helsinki 2000. http://ethesis.helsinki.fi/ julkaisut/mat/farma/vk/westermarck/useofmer.pdf.

10. Allen T. Particle size measurement. New York: Chapman \& Hall; 1997. p. 251

11. Morishige K. Adsorption hysteresis in ordered mesoporous silicas. Adsorption. 2008;14(2-3):157-63.

12. Liu H, Zhang L, Seaton NA. Sorption hysteresis as a probe of pore structure. Langmuir. 1993;9(10):2576-82.

13. Groen JC, Peffer LAA, Pérez-Ramírez J. Pore size determination in modified micro- and mesoporous materials. Pitfalls and limitations in gas adsorption data analysis. Microporous Mesoporous Mater. 2003;60(1-3):1-17.

14. Kloetstra KR, Zandbergen HW, van Koten MA, van Bekkum H. Thermoporometry as a new tool in analyzing mesoporous MCM41 materials. Catal Lett. 1995;33:145-56.

15. Brun M, Lallemand A, Quinson J, Eyraud C. A new method for the simultaneous determination of the size and shape of pores: the thermoporometry. Thermochim Acta. 1977;21:59-88.

16. Kunh W, Peterli E, Majer H. Freezing point depression of gels produced by high polymer network. J Polym Sci A. 1995;16(82):539-48.

17. Dessources AH, Hartmann S, Baba M, Huesing M, Nedelec JM. Multiscale characterization of hierarchically organized porous hybrid materials. J Mater Chem. 2012;22:2713-20.

18. Wulff M. Pore size determination by thermoporometry using acetonitrile. Thermochim Acta. 2004;419:291-4.

19. Husar B, Commereuc S, Lukac I, Chmela S, Nedelec JM, Baba M. Carbon tetrachloride as a thermoporometry liquid probe to study the cross-linking of styrene copolymer networks. J Phys Chem B. 2006;110:5315-20.

20. Nedelec JM, Grolier JPE, Baba M. Thermoporosimetry: a powerful tool to study the cross-linking in gels networks. J Sol-Gel Sci Technol. 2006;40:191-200.

21. Majda D, Makowski W, Mańko M, Mlekodaj K, Michalik-Zym A, Napruszewska BD, Zimowska M, Serwicka EM. Porosity characterization of SBA-15 silicas with thermoporosimetry of water and n-alkanes-the effect of the probe liquid nature. Microporous Mesoporous Mater. 2015;201:141-50.

22. Majda D, Korzeniowska A, Makowski W, Michalik-Zym A, Napruszewska BD, Zimowska M, Serwicka EM. Thermoporosimetry of $n$-alkanes for characterization of mesoporous SBA-15 silicas-refinement of methodology. Microporous Mesoporous Mater. 2016;222:33-43.

23. Majda D, Makowski W, Mańko M. Pore size distribution of micelle-templated silicas studied by thermoporosimetry using water and n-heptane. J Therm Anal Calorim. 2012;109:663-9.

24. Landry M. Thermoporometry by differential scanning calorimetry: experimental considerations and applications. Thermochim Acta. 2005;433:27-50.

25. Schmidt R, Hansen EW, Stocker M, Akporiaye D, Ellestad OH. Pore size determination of MCM-41 mesoporous materials by means of $1 \mathrm{H}$ NMR spectroscopy, $\mathrm{N}_{2}$ adsorption, and HREM. A preliminary study. J Am Chem Soc. 1995;117:4049-56.

26. Ishikiriyama $\mathrm{K}$, Todoki M. Evaluation of water in silica pores using differential scanning calorimetry. Thermochim Acta. 1995;256:213-26.

27. Schreiber A, Ketelsen I, Findenegg GH. Melting and freezing of water in ordered mesoporous silica materials. Phys Chem Chem Phys. 2001;3:1185-95.

28. Silvestre-Albero A, Jardim EO, Bruijn E, Meynen V, Cool P, Sepulveda-Escribano A, Silvestre-Albero J, Rodriguez-Reinoso F. Is there any microporosity in ordered mesoporous silicas? Langmuir. 2009;25:939-43. 
29. Ravikovitch PI, Neimark AV. Characterization of nanoporous materials from adsorption and desorption isotherms. Colloids Surf A. 2001;187:11-21.

30. Llewellyn PL, Schuth F, Grillet Y, Rouquero F, Rouquero J, Unger KK. Water sorption on mesoporous aluminosilicate MCM 41. Langmuir. 1995;11:574-7.

31. Ng E-P, Mintova S. Nanoporous materials with enhanced hydrophilicity and high water sorption capacity. Microporous Mesoporous Mater. 2008;114:1-26.

32. Pantalei C, Senesi R, Andreani C, Sozzani P, Comotti A, Bracco S, Beretta M, Sokola PE, Reiterd G. Interaction of single water molecules with silanols in mesoporous silica. Phys Chem Chem Phys. 2011;13:6022-8.

33. Nonaka A, Ishizaka EJ. Stepwise multilayer adsorption of water vapor on silica at temperatures from 130 to $150{ }^{\circ} \mathrm{C}$. J Colloid Interface Sci. 1977;63:381-8.

34. Whalen JW. Thermodynamic properties of water adsorbed on quartz. J Phys Chem. 1961;65:1676-81.

35. Bolis V, Busco C, Ugliengo P. Thermodynamic study of water adsorption in high-silica zeolites. J Phys Chem B. 2006;110: 14849-59.

36. Yang J, Meng S, Xu L, Wang EG. Water adsorption on hydroxylated silica surfaces studied using the density functional theory. Phys Rev B. 2005;71:035413.
37. Cauvel A, Brunel D, Renzo FD, Garrone E, Fubini B. Hydrophobic and hydrophilic behaviour of micelle templated mesoporous silica. Langmuir. 1997;13:2773-8.

38. Fubini B, Bolis V, Cavenago A, Garrone E, Ugleingo P. Structural and induced heterogeneity at the surface of some $\mathrm{SiO}_{2}$ polymorphs from the enthalpy of adsorption of various molecules. Langmuir. 1993;9(10):2712-20.

39. Majda D, Tarach K, Góra-Marek K, Michalik-Zym A, Napruszewska BD, Zimowska M, Serwicka EM. Thermoporosimetry of n-alkanes - towards deeper understanding the effect of the probe liquid nature. Microporous Mesoporous Mater. 2016;226:25-33.

40. Findenegg GH, Jahnert S, Akcakayiran D, Schreiber A. Freezing and melting of water confined in silica nanopores. ChemPhysChem. 2008;9:2651-9.

41. Ishikiriyama K, Todoki M, Motomura K. Pore size distribution measurements of silica gels by means of differential scanning calorimetry. Optimization for determination of PSD. J Colloid Interface Sci. 1995;171:92-102.

42. Billamboz N, Baba M, Grivet M, Nedelec J-MJ. A general law for predictive use of thermoporosimetry as a tool for the determination of textural properties of divided media. Phys Chem B. 2004;108:12032-7. 